\title{
The Mechanism of Heme Transfer from the Cytoplasmic Heme Binding Protein PhuS to the $\delta$-regioselective Heme Oxygenase of Pseudomonas aeruginosa
}

\author{
Mehul N. Bhakta and Angela Wilks* \\ Department of Pharmaceutical Sciences, School of Pharmacy, University of Maryland, 20 Penn \\ Street, Baltimore, MD 21201
}

\section{Abstract}

The opportunistic pathogen Pseudomonas aeruginosa has evolved two outer membrane receptor mediated uptake systems (encoded by the phu and has operons) by which it can utilize the hosts heme and hemeproteins as a source of iron. PhuS is a cytoplasmic heme binding protein encoded within the $p h u$ operon, and has previously been shown to function in the trafficking of heme to the ironregulated heme oxygenase ( $p a-\mathrm{HO})$. While the heme association rate for PhuS was similar to that of myoglobin, a markedly higher rate of heme dissociation $\left(\sim 10^{5} \mathrm{~s}^{-1}\right)$ was observed, in keeping with a function in heme-trafficking. Additionally, the transfer of heme from PhuS to $p a-\mathrm{HO}$ was shown to be specific and unidirectional when compared to transfer to the non-iron regulated heme oxygenase $(\mathrm{BphO})$, in which heme distribution between the two proteins merely reflects their relative intrinsic affinities for heme. Furthermore, the rate of transfer of heme from holo-PhuS to $p a-\mathrm{HO}$ of $0.11 \pm$ $0.01 \mathrm{~s}^{-1}$ is 30 -fold faster than that to apo-myoglobin, despite the significant higher binding affinity of apo-myoglobin for heme $\left(k_{\mathrm{H}}=1.3 \times 10^{-8} \mu \mathrm{M}\right)$ than that of PhuS $(0.2 \mu \mathrm{M})$. This data suggests that heme transfer to $p a-\mathrm{HO}$ is independent of heme affinity and is consistent with temperature dependence studies which indicate the reaction is driven by a negative entropic contribution, typical of an ordered transition state, and supports the notion that heme transfer from PhuS to $p a-\mathrm{HO}$ is mediated via a specific protein-protein interaction. In addition, $\mathrm{pH}$ studies, and reactions conducted in the presence of cyanide, suggest the involvement of spin transition during the heme transfer process, whereby the heme undergoes spin change from 6-c LS to 6-c HS either in PhuS or pa-HO. Based on the magnitudes of the activation parameters obtained in the presence of cyanide, whereby both complexes are maintained in a 6-c LS state, and the biphasic kinetics of heme transfer from holo-PhuS to $p a-H O-w t$, supports the notion that the spin-state crossover occur within holo-PhuS prior to heme transfer step. Alternatively, the lack of the biphasic kinetic with $p a-\mathrm{HO}-\mathrm{G} 125 \mathrm{~V}, 6-\mathrm{c}$ LS, and with comparable rate of heme transfer as $p a$-HO-is supportive of mechanism in which the spin-change could occur within $p a-\mathrm{HO}$. The present data suggests either or both of the two pathways proposed for heme transfer may occur under the present experimental conditions. The dissection of which pathway is physiologically relevant is the focus of ongoing studies.

Heme, a cofactor of proteins involved in a variety of biological processes such as oxygen transport and storage, oxygenation reactions, electron transfer and transcriptional regulation is also a redox-reactive, hydrophobic iron chelate that readily associates with membranes, and is

\footnotetext{
*Address correspondence to: Angela Wilks Department of Pharmaceutical Sciences School of Pharmacy University of Maryland 20 Penn Street, Baltimore, MD 21201 Tel. 410 706-2537 Fax.410 706-5017 e-mail: awilks@ rx.umaryland.edu.

Supporting information available: Temperature dependence of the rate constant of heme-transfer from PhuS to $p a-\mathrm{HO}-\mathrm{wt}, p a-\mathrm{HO}$ mutants and BphO. Table S2. Temperature dependence of the rate constant of heme-transfer from PhuS to $p a-\mathrm{HO}$-wt, $p a$-HO mutants and $\mathrm{BphO}$ in the presence of $10 \mathrm{mM} \mathrm{KCN}$.
} 
toxic to cells due to its ability to generate reactive oxygen species. Therefore, aerobic organisms have developed strategies to protect themselves from the harmful effects of "free" heme by sequestering it within specific proteins $(1,2)$. While hemeproteins serve a variety of biological functions very little is understood on the transport and shuttling of heme within cells.

Furthermore, heme has been shown to be a source of iron in numerous pathogenic bacteria and is required for survival and virulence $(1,2)$. Bacterial pathogens have developed sophisticated mechanisms by which they acquire heme directly from the host's hemeproteins or via a secreted hemophore that sequesters and returns heme to the outer-membrane receptor for internalization and further utilization (3-8). Once internalized heme is degraded by soluble heme oxygenases to biliverdin, $\mathrm{CO}$ and free iron and whereas the overall mechanism of heme degradation by bacterial HO's is fairly well understood (9), very little is known on how heme is transported within the bacterial cell.

The opportunistic pathogen Pseudomonas aeruginosa, a Gram negative bacterium, causes infections of immune compromised individuals, specifically cystic fibrosis patients and burn victims (10-12). In addition $P$. aeruginosa is rapidly becoming a leading cause of nosocomial infections in hospital and community settings. $P$. aeruginosa encodes two iron-regulated heme uptake operons, the Pseudomonas heme utilization ( $p h u)$ and heme assimilation system (has) (7). The $p h u$ operon encodes an outer membrane receptor (PhuR) and the cytoplasmic ATPase and permease proteins (Phu $\mathrm{U}$ and $\mathrm{V}$ ) which comprise an $\mathrm{ABC}$-transporter required for internalization of the heme. In addition, the $p h u$ operon encodes a periplasmic-binding protein (PhuT) a soluble receptor for the $\mathrm{ABC}$ transporter, and a cytoplasmic binding protein (PhuS) whose function is not well understood (7). In contrast, the has operon encodes the outermembrane receptor (HasR) a secreted hemophore (HasA) and the ATP-ase/permease (HasU and $\mathrm{V}$ ) required for secretion of the hemophore (13). While many of the proteins have either been characterized or have proposed functions based on similarity to the well characterized iron-siderophore uptake proteins, the cytoplasmic heme binding protein $(\mathrm{PhuS})$ were proposed to be heme oxygenases. This hypothesis was based on early genetic studies in which the phuS gene homolog hemS of Yersinia entercolitica on deletion showed heme toxicity when heme was given as the sole source of iron (14). We have recently characterized the heme binding protein, PhuS, in Pseudomonas aeruginosa as a heme-chaperone to the previously characterized iron-regulated heme oxygenase, $p a-\mathrm{HO}(15)$. More recently a second heme oxygenase $\mathrm{BphO}$ was characterized and in contrast to $p a-\mathrm{HO}$ it is not iron-regulated and yields biliverdin IX $\alpha$ as the product of the reaction, and not the $\delta$-regioselective isomer as for $p a-\mathrm{HO}$. The $\alpha$-biliverdin chromophore is transferred to the receptor protein (BphP) of a two-component sensor kinase for which the downstream function remains unknown $(16,17)$. These recent findings further confirmed the role of the iron-regulated $p a-\mathrm{HO}$ is solely in the mining of iron, suggesting that transfer of heme from PhuS is specific to the iron-regulated $p a-\mathrm{HO}$.

Initial spectroscopic characterization of the heme-PhuS complex at neutral $\mathrm{pH}$ indicates the heme to be predominantly six coordinate low spin (6-c LS) (15). However, heme-pa-HO at neutral $\mathrm{pH}$ has a 6-c HS heme, therefore, we hypothesize that heme transfer from PhuS to $p a-$ $\mathrm{HO}$ involves a switch in both the axial heme ligand and a change in the heme spin-state. In the present study, we have further characterized PhuS as a specific heme transfer protein to $p a$ $\mathrm{HO}$ based on competitive studies carried out with $p a-\mathrm{HO}$, selected $p a-\mathrm{HO}$-mutants with either an altered heme seating or spin-state, $\mathrm{BphO}$ the non-iron regulated $\mathrm{HO}$, myoglobin, and bovine serum albumin (BSA). In addition the mechanism of heme transfer from PhuS to $p a-\mathrm{HO}$ was investigated by measuring the kinetics of this process at several temperatures in the presence and absence of $\mathrm{CN}^{-}$in order to provide a better understanding of the heme transfer pathway. Taken together the data indicates that PhuS transfers heme specifically to $p a-\mathrm{HO}$, and a switch in spin-state from low-spin to high-spin occurs either in PhuS or $p a-\mathrm{HO}$ during the heme transfer. 


\section{Materials and Methods}

\section{Materials}

Hemin, myoglobin and bovine serum albumin (BSA) were purchased from Sigma-Aldrich. All other chemicals and reagents purchased were ACS reagent grade or higher. Heme solutions were prepared by dissolving heme in $0.1 \mathrm{~N}$ sodium hydroxide and buffering with $20 \mathrm{mM}$ Tris (pH 7.5) unless otherwise stated. Cyanoferric complexes of holo-PhuS and holo $p a$-HO were generated by the addition of excess amount of KCN $(10 \mathrm{mM})$ [CAUTION: Addition of acid to solutions containing cyanide can generate poisonous $\mathrm{HCN}$ gas].

\section{Purification and preparation of proteins}

Apomyoglobin was prepared using the methyl ethyl ketone method described by Ascoli $e$ t. al. (18). After hemin extraction with methyl ethyl ketone at acidic $\mathrm{pH}$, apo-globin was dialyzed extensively against $20 \mathrm{mM}$ Tris, $\mathrm{pH} 7.5$. The apoprotein was then centrifuged to remove any remaining precipitate and concentrated to approximately $0.5 \mathrm{mM}$ using an $\varepsilon_{280}$ of 15.2 $\mathrm{mM}^{-1} \mathrm{~cm}^{-1}$ and stored at $-80^{\circ} \mathrm{C}$. The PhuS, $p a$-HO-wt, $p a$-HO-mutants and $\mathrm{BphO}$ proteins were expressed and purified as described previously $(15,19)$.

\section{Heme Transfer Experiments}

All heme transfer kinetic experiments were carried out with an Applied Photophysic stopped flow spectrometer (model SX.18MV) unless otherwise stated. Sample preparation was carried out as previously reported previously (15). In brief, components of the reactions were mixed and pre-incubated for $5 \mathrm{~min}$ at the appropriate temperature. Heme transfer studies were conducted in the presence $10 \mu \mathrm{M}$ PhuS and $30 \mu \mathrm{M} p a-\mathrm{HO}$ in $20 \mathrm{mM}$ Tris, $\mathrm{pH} 7.5$, unless otherwise stated and were monitored at 405 and $419 \mathrm{~nm}$ in the absence and presence of $10 \mathrm{mM}$ $\mathrm{CN}^{-}$, respectively.

Heme transfer experiments from holo-PhuS to apo $p a-\mathrm{HO}$, apo-myoglobin or BSA were followed by UV-visible spectrometry, and full spectra were collected as a function of time. The absorbance changes at the appropriate wavelength were fitted to either a one (eq. 1) or a two-exponential decay (eq. 2) where $k_{1}$ and $k_{2}$ are the observed rate constants for the fast and slow phases, respectively. $A_{1}$ and $A_{2}$ are related to the initial absorbances, and $A_{t}$ is the absorbance at time t. The calculated $k_{\text {obs }}$ for each reaction were determined from the average of at least three measurements. Non-linear curve fitting of the data was performed with the supplied Applied Photophysics software and Sigma-Plot.

$$
\begin{gathered}
A_{t}=A_{0}+A_{1}\left(1-e^{-k_{1}{ }^{t}}\right) \\
A_{t}=A_{0}+A_{1}\left(1-e^{-k}{ }_{1}{ }^{t}\right)+A_{2}\left(1-e^{-k_{2}{ }^{t}}\right)
\end{gathered}
$$

The extent of heme transfer from the heme-PhuS complex to $p a-\mathrm{HO}, \mathrm{BphO}$ and BSA was verified by UV-visible absorbance ( $406 \mathrm{~nm}$ for heme, $280 \mathrm{~nm}$ for protein) and SDS-PAGE analysis of chromatographic fractions separated from the reaction mixture by gel filtration (Sepharose S-100, $1.5 \times 120$-cm column, equilibrated with $20 \mathrm{mM}$ Tris- $\mathrm{HCl}(\mathrm{pH} 8.0), 100 \mathrm{mM}$ $\mathrm{NaCl})$. 


\section{Temperature-dependence analysis}

The natural log of the rate constants for each averaged set of experimental data were plotted against the reciprocal of the absolute temperature. The data were then fit to the Arrhenius equation (eq. 3) using the linear fitting function in the plotting program sigma plot. In this equation, $\mathrm{A}$ is the Arrhenius pre-exponential factor and $\mathrm{R}$ is the gas constant. The activation enthalpy and activation entropy and Gibbs free energy of heme transfer were determined using eqs 4, 5 and 6, respectively, where $k_{\mathrm{B}}$ is the Boltzmann constant and $h$ is Planck's constant.

$$
\mathrm{Ln} \mathrm{k}=\ln A-\mathrm{E}_{\mathrm{a}} / \mathrm{RT}
$$

$$
\Delta \mathrm{S}^{\ddagger}=\mathrm{R}\left(\ln A-\ln \left(k_{\mathrm{B}} \mathrm{T} / h\right)\right)
$$

$$
\Delta \mathrm{G}^{\ddagger}=\Delta \mathrm{H}^{\ddagger} \quad-\mathrm{T} \Delta \mathrm{S}^{\ddagger}
$$

\section{Results}

\section{Heme binding to PhuS and pa-HO}

We have previously shown that both PhuS and $p a-\mathrm{HO}$ bind one heme per monomer with similar affinities of 0.6 and $0.2 \mu \mathrm{M}$ for $p a-\mathrm{HO}$ and PhuS, respectively (15). The kinetics of the reactions were investigated by mixing in a stopped-flow apparatus $2 \mu \mathrm{M}$ heme with either PhuS or $p a-$ $\mathrm{HO}$ at concentrations between 20 to $100 \mu \mathrm{M}$ of apo-protein, and monitored at either 410 or 405 $\mathrm{nm}$, respectively. Heme solutions were used at a low concentration to minimize the tendency to form $\mu$-oxo-dimers. As shown in Table I, association rate constants of $1.8 \pm 0.1 \times 10^{5}$ and $1.1 \pm 0.1 \times 10^{5} \mathrm{M}^{-1} \mathrm{~s}^{-1}$ were obtained for heme binding to PhuS or $p a-\mathrm{HO}$, respectively. The heme dissociation rate constants $\left(k_{-\mathrm{H}}\right)$ calculated from the average heme affinity $\left(\mathrm{K}_{\mathrm{d}}\right)$ and the association rate constants $\left(k_{\mathrm{H}}\right)$ for PhuS or $p a-\mathrm{HO}$ were 0.036 and $0.066 \mathrm{~s}^{-1}$, respectively. The rate of heme association of PhuS or $p a-\mathrm{HO}$ is similar to BSA (20) and comparable to that of the myoglobin (21), yet, both PhuS and $p a$-HO have a markedly higher rate of heme dissociation compared to either myoglobin $\left(8.4 \times 10^{-7} \mathrm{~s}^{-1}\right)$ or BSA $\left(3.2 \times 10^{-4} \mathrm{~s}^{-1}\right)$, which would be a requirement of a heme-trafficking protein.

\section{Heme Transfer Experiments}

Kinetic traces for the heme transfer from PhuS to $p a$-HO-wt, mutants with altered regioselectivity ( $p a-\mathrm{HO}-\mathrm{N} 19 \mathrm{~K} / \mathrm{F} 117 \mathrm{Y}$ ( $p a-\mathrm{HO}-\mathrm{DM}), p a-\mathrm{HO}-\mathrm{N} 19 \mathrm{~K} / \mathrm{F} 117 \mathrm{Y} / \mathrm{K} 34 \mathrm{~N}$ (DMK34N), $p a-\mathrm{HO}-\mathrm{N} 19 \mathrm{~K} / \mathrm{F} 117 \mathrm{Y} / \mathrm{K} 132 \mathrm{~A}(\mathrm{DM}-\mathrm{K} 132 \mathrm{~A})$ ), the altered spin-state mutant ( $p a-$ HO-G125V) or BphO are shown in Figure 1. At pH 7.5, heme transfer from PhuS to $p a-\mathrm{HO}-$ wt, the $p a-\mathrm{HO}$ mutants, or BphO clearly display biphasic kinetics and the time courses were therefore fit to a two-exponential expression. The average rates of the initial kinetic phases and the slow phase were $0.10 \mathrm{~s}^{-1}$ and $0.01 \mathrm{~s}^{-1}$ at $25^{\circ} \mathrm{C}(\mathrm{pH}$ 7.5) respectively. In contrast, the heme transfer from heme-PhuS to $p a-\mathrm{HO}-\mathrm{G} 125 \mathrm{~V}$ displayed single phase kinetics and was fit to a single-exponential expression with a $k_{\mathrm{obs}}$ of $0.1 \mathrm{~s}^{-1}$. Therefore, we attribute the initial kinetic phase, $k_{1}$, to heme transfer from holo-PhuS to the respective HO proteins and the second phase 
rate, $k_{2}$, to change in heme spin change from LS to HS based on the results obtained with $p a$ HO-wt and $p a-\mathrm{HO}-\mathrm{G} 125 \mathrm{~V}$.

The $p a-\mathrm{HO}-\mathrm{DM}, \mathrm{DM}-\mathrm{K} 34 \mathrm{~N}$, and DM-K132A, were previously constructed and characterized to determine the role of specific surface residues in coordination of the heme propionates in stabilizing the heme for regioselective oxidative cleavage. These mutations have previously been shown to destabilize the heme within the protein such that it is in dynamic in-plane rotation between two seatings that yield an altered isomer pattern than that of the wild-type protein $(19,22)$. Therefore we would predict that such a destabilization would have an effect on the rate of heme transfer. Surprisingly, these mutants did not greatly alter or affect the overall rate of heme transfer, $k_{1}$, from PhuS when compared to the wild type $p a-\mathrm{HO}$, although a 3 -fold decrease in the rate of the slow phase, $k_{2}$, was observed. This data suggests that amino acids that stabilize the heme within $p a-\mathrm{HO}$ have a minor influence on the overall heme transfer process.

The rate of heme transfer, $k_{1}$, from PhuS to the $p a$-HO mutants and BphO decreased by 3 -fold going from $\mathrm{pH} 6.5$ to 8.5 (Table II). The increased in the rate of heme transfer to $p a-\mathrm{HO}$ and $\mathrm{BphO}$ is most likely due to the protonation of the proximal His side chain of PhuS, facilitating the loss (dissociation) of the Fe-His bond (23-25). Although, no correlation was observed between $\mathrm{pH}$ and the second phase of the reaction, at high $\mathrm{pH}$ the slow phase, $k_{2}$, seemingly disappears, giving rise to a monophasic kinetics as shown in Figure 2. Previous spectroscopic characterization of both $p a-\mathrm{HO}$ and PhuS indicates that at neutral $\mathrm{pH}(\mathrm{pH} 7.5)$ the heme-PhuS and heme-pa-HO complexes are 6-c LS and 6-c HS, respectively $(15,26)$. However, above pH 8.0 an alkaline transition occurs whereby $p a-\mathrm{HO}$ switches from a 6-c HS to a 6-c LS system, indicating that a spin-transition may be involved in the transfer of heme from PhuS to $p a-\mathrm{HO}$ or BphO.

In order to test this hypothesis, experiments were conducted in the presence of $10 \mathrm{mM} \mathrm{CN}^{-}$. Treatment of holo-PhuS with $\mathrm{CN}^{-}$at $\mathrm{pH} 7.5$ resulted in a shift of the Soret peak to $419 \mathrm{~nm}$ and a replacement of the distinct 528 - and $560 \mathrm{~nm}$ peaks with a broad band at $530 \mathrm{~nm}$, indicative of a low-spin CN-complex. As shown in Figure 1, the absorbance time course for heme transfer from PhuS to $p a-\mathrm{HO}-\mathrm{wt}$, mutants or BphO, displays biphasic kinetics. Although, the rate of the fast phase, $k_{1}$ of $0.12 \mathrm{~s}^{-1}$, was similar to the rate observed in the absence of $\mathrm{CN}^{-}$, the rate of the slow phase, $k_{2}$ of $0.03 \mathrm{~s}^{-1}$, was 3 -fold slower compared to the rate observed for the slow phase in the absence of $\mathrm{CN}^{-}$. Inhibition of $k$ by $\mathrm{CN}^{2}$ is indicative and supportive of the involvement of spin-change during the heme transfer.

\section{Effect of Temperature on the rate of Heme Transfer}

The temperature dependence of heme transfer from PhuS to $p a-\mathrm{HO}$, the $p a-\mathrm{HO}-$ mutants or $\mathrm{BphO}$ at $\mathrm{pH} 7.5$ are shown in Table III. The rate of heme transfer increased with increasing temperature (Supplementary Information; Table S1), and displayed linear and non-linear Arrhenius plots for the fast-, $k_{1}$, and the slow-phase, $k_{2}$, of heme transfer from PhuS, respectively (Figure 3 ). A concave Arrhenius plot was obtained for the slow-phase, $k_{2}$, of the reaction over the complete temperature range, and is most likely due to composite of other rate constants or steps.

The activation energies at $\mathrm{pH} 7.5$ for the heme transfer from PhuS to $p a-\mathrm{HO}-\mathrm{wt}, p a-\mathrm{HO}$ mutants and $\mathrm{BphO}$ range from $\sim 12$ to $14 \mathrm{kcal} / \mathrm{mol}$. The activation enthalpy and entropy ranged from $11.3-13.7 \mathrm{kcal} / \mathrm{mol}$ and -15 to $-23 \mathrm{cal} \mathrm{mol}^{-1} \mathrm{~K}^{-1}$, respectively (Table III). The higher activation enthalpy of heme transfer to $\mathrm{BphO}$ by $\sim 2 \mathrm{kcal} / \mathrm{mol}$ compared to $p a-\mathrm{HO}$, suggests that heme transfer from PhuS to $p a-\mathrm{HO}$ is more favorable than to BphO. However, the increase in enthalpy with $\mathrm{BphO}$ appears to be compensated by larger and favorable entropy, since the free energy of activation is similar for both proteins. 
The rates of heme transfer in the presence of $\mathrm{CN}^{-}$and as a function of temperature are provided in the Supplementary Information (Table S2). The temperature dependence of the activation parameters for heme transfer in the presence of $\mathrm{CN}^{-}$are summarized in Table IV. The activation energy ranged from $12.3-17.2 \mathrm{kcal} / \mathrm{mol}$ for the $p a-\mathrm{HO}-\mathrm{wt}$ and $p a-\mathrm{HO}$ mutants, and $12.8 \mathrm{kcal} /$ $\mathrm{mol}$ for $\mathrm{BphO}$ as calculated from the slopes of Arrhenius plots. The activation enthalpy for some of the $p a$-HO-mutants drifts considerably from the WT protein and indicates that these amino acids must play an important role during the heme transfer process. The increase in the activation enthalpy appears to be compensated by favorable (or unfavorable) activation entropy. The high activation enthalpy obtained in the presence of $\mathrm{CN}^{-}$(compared to without $\mathrm{CN}^{-}$) of $\sim 12 \mathrm{kcal} / \mathrm{mol}$ suggests that $p a-\mathrm{HO}$ is not energetically competent to receive heme and/ or stay in a low-spin-heme conformation at $\mathrm{pH}$ 7.5. On the other hand, transfer to BphO seems to be favored and the transition state for the heme transfer is highly organized in the presence of $\mathrm{CN}^{-}$. This may imply that the transfer to $\mathrm{BphO}$ as previously shown is not facilitated by a direct protein-protein interaction as is that of $p a-\mathrm{HO}$, and the $\mathrm{CN}^{-}$complex effectively destabilizes the holo-PhuS.

\section{Heme Transfer from PhuS and pa-HO to Myoglobin or Bovine Serum Albumin (BSA)}

The rate of heme-transfer from PhuS or $p a-\mathrm{HO}$ to myoglobin was examined as described in the experimental section. The time course for the transfer of heme from $p a-\mathrm{HO}$ or PhuS to myoglobin was fit to a single exponential (Figure 4). The pseudo-first order rate constant for the heme dissociation from $p a$-HO and PhuS were $3.5 \pm 0.1 \times 10^{-3} \mathrm{~s}^{-1}$ and $3.8 \pm 0.1 \times 10^{-3}$ $\mathrm{s}^{-1}$, respectively. The relative high rate of heme dissociation compared to that for myoglobin (Table I), suggests that heme binds very weakly to PhuS and $p a-\mathrm{HO}$, which would be expected for proteins that are involved heme-trafficking and degradation, respectively. The 30-fold decrease in the rate of heme transfer from PhuS and $p a-\mathrm{HO}$ to myoglobin suggests that this process is solution mediated as result of the high affinity of myoglobin for heme and not via a direct protein interaction. In order to further support the above hypothesis; reactions were conducted with BSA, which has a similar rate of heme association and dissociation as $p a-\mathrm{HO}$. Under standard experimental conditions, no heme transfer from PhuS to BSA occurred, as judged by UV-visible spectroscopy and size-exclusion chromatography (data not shown).

\section{Discussion}

It has previously been shown that the PhuS homolog HemS is required for efficient heme utilization in $Y$. entercolitica (14). We therefore hypothesized that heme as it enters the cell is sequestered by PhuS which serves as a heme carrier that delivers heme to the iron-regulated heme oxygenase, $p a-\mathrm{HO}$. Oxidative ring opening of the porphyrin by $p a-\mathrm{HO}$ then releases iron for further utilization by the cell. Recently, we provided evidence for this hypothesis via a comprehensive in vitro biochemical and spectroscopic analysis of the heme-PhuS complex and its role as a specific heme chaperone to $p a-\mathrm{HO}$ (15). The possibility of non-specific solution mediated heme transfer was judged unlikely since similar kinetics were observed under excess amounts of $p a-\mathrm{HO}$. Furthermore, a similar product distribution and regioselectivity was observed on coupled oxidation of either $p a-\mathrm{HO}$ alone or Phus/pa-HO, which suggests that heme transfer from PhuS to $p a$-HO is specific and most likely driven by direct protein-protein interaction (data not shown). The inability of apo-PhuS to acquire heme from holo-pa-HO, also excluded the possibility of reverse heme transfer and confirmed that the transfer is unidirectional and facilitated by direct protein-protein interaction, which was further confirmed by surface plasmon resonance (SPR) (15).

In contrast, heme transfer from holo-PhuS to $\mathrm{BphO}$ is reversible indicating that the affinity of heme-PhuS for $\mathrm{BphO}$ is low and the absorbance spectra recorded during transfer do not exhibit behavior consistent with complete transfer of the heme. This behavior is indicative of a 
dissociative heme transfer mechanism that involves release of the heme into solution which was confirmed by the lack of a protein-protein interaction as judged by SPR. Therefore, in contrast to the mechanistically specific transfer of heme from PhuS to $p a-\mathrm{HO}$, the transfer of heme between PhuS and BphO is most likely governed by their relative intrinsic affinities for heme.

The rapid and unidirectional transfer of heme from PhuS to $p a-\mathrm{HO}$, and the high affinity of PhuS for $p a-\mathrm{HO}$ compared to BphO, supports the hypothesis that PhuS acts as heme-chaperone to $p a-H O$. In order to further substantiate this, and to show that heme transfer is not governed by the intrinsic heme-affinity of the respective proteins, transfer studies from holo-PhuS to $p a-\mathrm{HO}$ were conducted in parallel with myoglobin and BSA, which have a higher heme affinity than either $p a-\mathrm{HO}$ or PhuS. If the intrinsic heme affinity of a protein is the primary factor, then the rate of heme transfer from holo-PhuS to BSA and apo-myoglobin should have been similar or decidedly faster, respectively, than that of transfer to $p a-H O$. However, the lack of heme transfer observed for BSA, and a slow rate for myoglobin indicates that heme transfer to $p a$ $\mathrm{HO}$ is independent of the heme affinity of the protein, and other factors such as a proteinprotein interaction may be involved in triggering heme transfer.

The rate of heme transfer from PhuS to $p a-\mathrm{HO}$ and the $p a-\mathrm{HO}$ mutants is similar regardless of $\mathrm{pH}$ (Table II), although the increase in the rate of heme transfer at acidic $\mathrm{pH}$ may be due to protonation of the proximal histidine (23-25). However, the rate of heme transfer appears to be temperature dependent with increased rates at higher temperatures. The increase in the activation energy for heme transfer from holo-PhuS to the $p a-\mathrm{HO}$ mutants relative to the native protein is most likely due to the loss of favorable binding or interaction between PhuS and $p a$-HO. The $p a-\mathrm{HO}$ mutants introduced heme propionate interactions required for $\alpha$ regioselective specificity ( $p a-\mathrm{HO}-\mathrm{N} 19 \mathrm{~K} / \mathrm{F} 117 \mathrm{Y})$ as in other characterized $\mathrm{HO}$ enzymes and additionally removed residues that stabilize the $\delta$-regioselective heme seating ( $p a-\mathrm{HO}-\mathrm{N} 19 \mathrm{~K}$ )

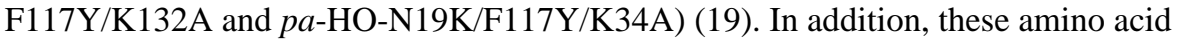
replacements are located on the heme binding face of $p a-\mathrm{HO}$ and, as well as stabilizing the bound heme, are located such that in the apo-pa-HO they may provide additional electrostatic or hydrogen-bonding interactions with the holo-PhuS (22). Furthermore, reactions conducted in the presence of $200 \mathrm{mM}$ sodium chloride inhibited the process by $\sim 40 \%$ (data not shown), suggesting that electrostatic interactions may play a significant role during heme transfer from PhuS to $p a$-HO. The low activation of entropy of the wild-type $p a$-HO suggests that the transition state is highly ordered, and this stabilization could be provided in the form of a protein-protein interaction, which is consistent with the gain in entropy observed for the $p a$ HO-mutants and BphO. However, an exact interpretation of these effects will require a more extensive study with a larger set of mutants at these and other positions in and around the heme pocket of both PhuS and pa-HO.

Previous observations suggested that at neutral $\mathrm{pH}$ the heme-PhuS and heme-pa-HO complexes are 6-c LS and 6-c HS, respectively $(15,19)$. Therefore, changes in both the spin-state and axial heme coordination must occur during the transfer reaction. These transformations may be driven by the free energy yield of protein-protein complexation, thereby triggering the transfer reaction. The ready accessibility of both the 6-c LS and 6-c HS spin-states in the heme-PhuS complex suggests this could be a feasible triggering mechanism for heme transfer. In the presence of a strong ligand such as $\mathrm{CN}^{-}$both holo-PhuS and holo-pa-HO are 6-c LS, as expected. Therefore, reactions conducted in the presence of $\mathrm{CN}^{-}$should have exhibited firstorder kinetic, since heme transfer from PhuS to $p a-\mathrm{HO}$ should not involve spin change prior (in PhuS) or after ( $p a-\mathrm{HO}$ ) heme transfer step. However, the biphasic kinetic observed in the presence of $\mathrm{CN}^{-}$suggest that heme-spin-change must occur within PhuS prior to the heme transfer step. 
Thermodynamic parameters obtained for the reaction conducted in the presence and absence $\mathrm{CN}^{-}$indicates that enthalpy and entropy greatly influence heme transfer. Although the free energy of activation $\Delta \mathrm{G}^{\ddagger}$ values for the heme transfer in the presence or absence of $\mathrm{CN}^{-}$are similar, $\Delta \mathrm{H}^{\ddagger}$ for heme transfer is higher $\sim 2 \mathrm{kcal} / \mathrm{mol}$ in the presence of $\mathrm{CN}^{-}$, while $\Delta \mathrm{S}^{\ddagger}$ is lower $\sim 8 \mathrm{calmol}^{-1} \mathrm{~K}^{-1}$ in the absence of $\mathrm{CN}^{-}$. Thus, the equal free energies of these two transition states are due to the compensating differences in enthalpy and entropy. The small negative relative activation enthalpy, $\Delta \Delta \mathrm{H}^{\dagger}$, determined for heme-transfer from holo-PhuS to $p a-\mathrm{HO}$ wt or the $p a-\mathrm{HO}$-mutants, suggests that the heme transfer in the absence of $\mathrm{CN}^{-}$is favored by activation enthalpy compared to the reaction in the presence of $\mathrm{CN}^{-}$. However, the relative activation entropy, $\Delta \Delta \mathrm{S}^{\ddagger}$, for the heme-transfer is negative, suggesting that the transition state for the heme-transfer in the absence of $\mathrm{CN}^{-}$is much more ordered, organized, and entropically unfavorable in comparison to that of in the presence of $\mathrm{CN}^{-}$. These results suggest that the transition states of the reaction in the presence and absence of $\mathrm{CN}^{-}$are structurally different and consequently the gain of entropy in the $\mathrm{CN}^{-}$dependent reaction could be attributed to major changes in conformation of the protein and/or the spin-state of the heme prior to the transfer. In addition, the high activation enthalpy associated with the $\mathrm{CN}^{-}$reaction suggests that PhuS is not primed to transfer heme in the low-spin state and must undergo a spin-transition prior to heme-transfer, as shown in Scheme I, pathway a. This pathway is further supported by the fact that reactions conducted in the presence of $\mathrm{CN}^{-}$and at $\mathrm{pH} 8.5$ should have behaved similarly to the $p a-H O G 125 \mathrm{~V}$ mutant, since both holo-PhuS and holo-pa-HO are 6-c LS. However, biphasic kinetic observed in the above mentioned reactions is suggestive of a spin-change occurring within the heme-PhuS complex, and this could be the triggering step that leads to heme transfer.

Since $k_{1}$ and $k_{2}$ are associated to the rate of heme transfer and spin-change respectively, then the formation of the PhuS(HS):pa-HO complex is rate determining $\left(k_{2}<k_{1}\right)$ and first-order kinetic should have been observed if only pathway a is operative. However, the presence of the biphasic kinetic can be rationalized if an alternate pathway is also operative which involves a direct transfer of heme from PhuS to $p a-H O$ (Scheme I, pathway b). The scenario of both pathways being operative is plausible whereby pathway a shows a first order reaction with a rate constant of $k_{2}$, and pathway b would compromise a first order transfer of heme from holoPhuS to $p a-\mathrm{HO}$, producing a transient holo-pa-HO (LS) species, followed by conversion to high spin state within $p a-\mathrm{HO}$. The concave Arrhenius plot for $k_{2}$ is consistent with the assumption that this pathway consists of at least two kinetic steps. This model explains the observed results with $\mathrm{pa}-\mathrm{HO}-\mathrm{G} 125 \mathrm{~V}, 6-\mathrm{c} \mathrm{LS}$, which has a similar rate of heme transfer, $k_{1}$ of $\sim 0.1 \mathrm{~s}^{-1}$, as the wild-type protein and supports the notion that spin-transition could occurs within $p a-\mathrm{HO}$ and suggest that heme spin crossover may not be required prior to the heme transfer step. Although, the data does not conclusively support the possibility of either or both pathways being operative, it clearly shows that spin change must be involved during the heme transfer from holo-PhuS to apo-pa-HO.

\section{Concluding Remarks}

Taken together these studies indicate that the cytoplasmic heme binding protein PhuS acts as a specific heme-chaperone to the iron-regulated $p a-\mathrm{HO}$, and the biphasic behavior associated with heme transfer leads us to propose a model which involves a dual pathway for heme transfer. On the basis of the magnitudes of the activation parameters for the reactions conducted in the presence and absence of $\mathrm{CN}^{-}$, we propose that spin-change should occur within the heme-PhuS complex, and this is the triggering step that leads to heme transfer. Results from $p a-\mathrm{HO}-\mathrm{G} 125 \mathrm{~V}$ suggest that spin-change may not be required, and is supportive of a pathway which involves a direct heme transfer from PhuS to $p a-\mathrm{HO}$. While the current data supports the requirement of a spin-state crossover for heme transfer from PhuS to $p a-H O$ the specific step in the reaction where this transition occurs will require further experimental analysis. 


\section{Supplementary Material}

Refer to Web version on PubMed Central for supplementary material.

\section{Acknowledgements}

This work was supported by National Institutes of Health Grant AI-48551

\section{Abbreviations}

pa-HO, Pseudomonas aeruginosa $\delta$-regioselective heme oxygenase; BphO, Pseudomonas aeruginosa $\alpha$-regioselective heme oxygenase; $\mathrm{CN}^{-}$, potassium cyanide solution, heme, protoporphyrin IX regardless of oxidation state; has, heme assimilation system; phu, Pseudomonas heme uptake; 6-c HS, six-coordinate high spin; 6-c LS, six-coordinate low spin..

\section{References}

1. Wandersman C, Stojiljkovic I. Bacterial heme sources: the role of heme, hemoprotein receptors and hemophores. Curr Opin Microbiol 2000;3:215-220. [PubMed: 10744995]

2. Wandersman C, Delepelaire P. Bacterial iron sources: from siderophores to hemophores. Annu Rev Microbiol 2004;58:611-47. [PubMed: 15487950]

3. Henderson DP, Payne SM. Cloning and characterization of the Vibrio cholerae genes encoding the utilization of iron from haemin and haemoglobin. Mol Microbiol 1993;7:461-9. [PubMed: 8384684]

4. Mills M, Payne SM. Genetics and regulation of heme iron transport in Shigella dysenteriae and detection of an analogous system in Escherichia coli O157:H7. J Bacteriol 1995;177:3004-9. [PubMed: 7768795]

5. O'Malley SM, Mouton SL, Occhino DA, Deanda MT, Rashidi JR, Fuson KL, Rashidi CE, Mora MY, Payne SM, Henderson DP. Comparison of the heme iron utilization systems of pathogenic Vibrios. J Bacteriol 1999;181:3594-8. [PubMed: 10348876]

6. Drazek ES, Hammack CA, Schmitt MP. Corynebacterium diphtheriae genes required for acquisition of iron from haemin and haemoglobin are homologous to ABC haemin transporters. Mol Microbiol 2000;36:68-84. [PubMed: 10760164][In Process Citation]

7. Ochsner UA, Johnson Z, Vasil ML. Genetics and regulation of two distinct haem-uptake systems, phu and has, in Pseudomonas aeruginosa. Microbiology 2000;146:185-98. [PubMed: 10658665]

8. Mey AR, Payne SM. Haem utilization in Vibrio cholerae involves multiple TonB-dependent haem receptors. Mol Microbiol 2001;42:835-49. [PubMed: 11722746]

9. Wilks A. Heme oxygenase: evolution, structure, and mechanism. Antioxid Redox Signal 2002;4:60314. [PubMed: 12230872]

10. Costerton JW. Cystic fibrosis pathogenesis and the role of biofilms in persistent infection. Trends Microbiol 2001;9:50-2. [PubMed: 11173226]

11. Currie AJ, Speert DP, Davidson DJ. Pseudomonas aeruginosa: role in the pathogenesis of the CF lung lesion. Semin Respir Crit Care Med 2003;24:671-80. [PubMed: 16088583]

12. Elkin S, Geddes D. Pseudomonal infection in cystic fibrosis: the battle continues. Expert Rev Anti Infect Ther 2003;1:609-18. [PubMed: 15482158]

13. Letoffe S, Ghigo JM, Wandersman C. Secretion of the Serratia marcescens HasA protein by an ABC transporter. J Bacteriol 1994;176:5372-7. [PubMed: 8071214]

14. Stojiljkovic I, Hantke K. Hemin uptake system of Yersinia enterocolitica: similarities with other TonB-dependent systems in gram-negative bacteria. Embo J 1992;11:4359-67. [PubMed: 1425573]

15. Lansky IB, Lukat-Rodgers GS, Block D, Rodgers KR, Ratliff M, Wilks A. The cytoplasmic hemebinding protein (PhuS) from the heme uptake system of Pseudomonas aeruginosa is an intracellular heme-trafficking protein to the delta -regioselective heme oxygenase. J Biol Chem 2006;281:1365262. [PubMed: 16533806]

16. Tasler R, Moises T, Frankenberg-Dinkel N. Biochemical and spectroscopic characterization of the bacterial phytochrome of Pseudomonas aeruginosa. Febs J 2005;272:1927-36. [PubMed: 15819886] 
17. Wegele R, Tasler R, Zeng Y, Rivera M, Frankenberg-Dinkel N. The heme oxygenase(s)-phytochrome system of Pseudomonas aeruginosa. J Biol Chem 2004;279:45791-802. [PubMed: 15310749]

18. Ascoli F, Fanelli MR, Antonini E. Preparation and properties of apohemoglobin and reconstituted hemoglobins. Methods in Enzymol 1981;76:72-87. [PubMed: 7329287]

19. Caignan GA, Deshmukh R, Wilks A, Zeng Y, Huang HW, Moenne-Loccoz P, Bunce RA, Eastman MA, Rivera M. Oxidation of heme to beta- and delta-biliverdin by Pseudomonas aeruginosa heme oxygenase as a consequence of an unusual seating of the heme. J Am Chem Soc 2002;124:1487992. [PubMed: 12475329]

20. Gattoni M, Boffi A, Sarti P, Chiancone E. Stability of the heme-globin linkage in ab dimers and isolated chains of human hemoglobin. A study of the heme transfer reaction from the immobilized proteins to albumin. J Biol Chem 1996;271:10130-6. [PubMed: 8626572]

21. Hargrove MS, Barrick D, Olson JS. The Association Rate Constant for Heme Binding to Globin Is Independent of Protein Structure. Biochemistry 1996;35:11293-99. [PubMed: 8784183]

22. Friedman J, Lad L, Li H, Wilks A, Poulos TL. Structural basis for novel delta-regioselective heme oxygenation in the opportunistic pathogen Pseudomonas aeruginosa. Biochemistry 2004;43:523945. [PubMed: 15122889]

23. Giacometti GM, Traylor TG, Ascenzi P, Brunori M, Antonini E. Reactivity of ferrous myoglobin at low pH. J Biol Chem 1977;252:7447-8. [PubMed: 21180]

24. Coletta M, Ascenzi P, Traylor TG, Brunori M. Kinetics of carbon monoxide binding to monomeric hemoproteins. Role of the proximal histidine. J Biol Chem 1985;260:4151-5. [PubMed: 3980472]

25. Hargrove MS, Singleton EW, Quillin ML, Ortiz LA, Phillips GN Jr. Olson JS, Mathews AJ. His64 (E7) -> Tyr apomyoglobin as a reagent for measuring rates of hemin dissociation. J Biol Chem 1994;269:4207-14. [PubMed: 8307983]

26. Caignan GA, Deshmukh R, Zeng Y, Wilks A, Bunce RA, Rivera M. The hydroxide complex of Pseudomonas aeruginosa heme oxygenase as a model of the low-spin iron(III) hydroperoxide intermediate in heme catabolism: 13C NMR spectroscopic studies suggest the active participation of the heme in macrocycle hydroxylation. J Am Chem Soc 2003;125:11842-52. [PubMed: 14505406]

27. Rodriguez JC, Wilks A, Rivera M. Backbone NMR assignments and H/D exchange studies on the ferric azide- and cyanide-inhibited forms of Pseudomonas aeruginosa heme oxygenase. Biochemistry 2006;45:4578-92. [PubMed: 16584193] 


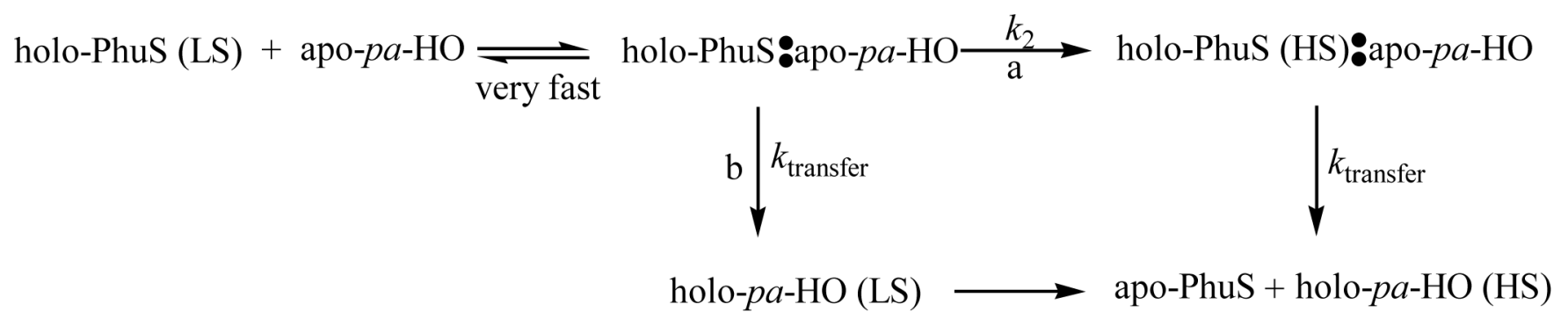

Scheme I. 


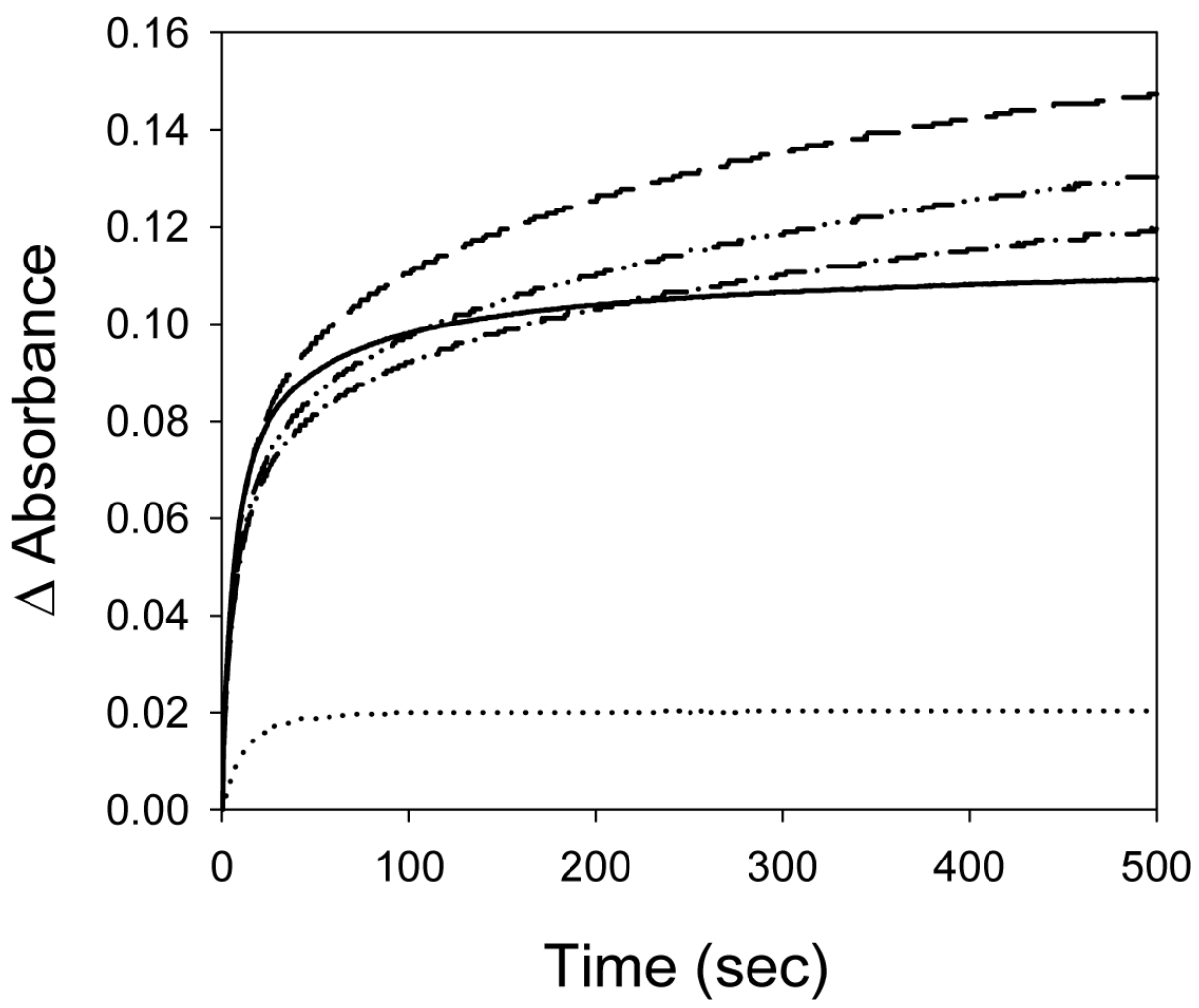

Figure 1.

Time course of Heme-Transfer from PhuS to pa-HO, paHO-mutants and BphO. (-) pa-HO (Wt); (- ) pa-HO-N19K/F117Y(DM); (-.-) pa-HO-DM-K132A; (-..-) pa-HO-DM-K34N;

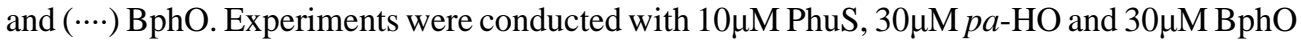
in $20 \mathrm{mM}$ Tris- $\mathrm{HCl}, \mathrm{pH} 7.5$, at $25^{\circ} \mathrm{C}$, and time course were measured at $405 \mathrm{~nm}$. 


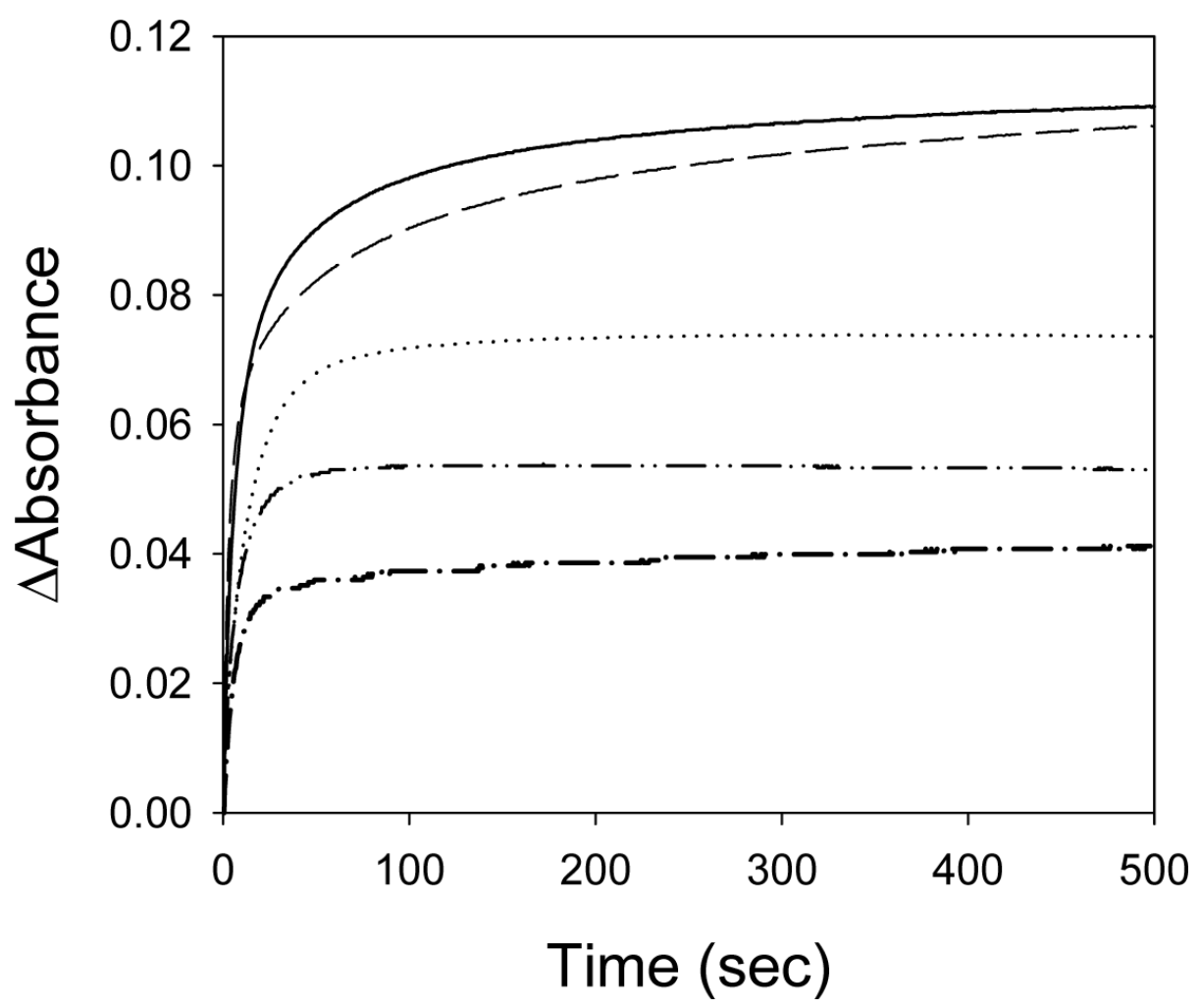

Figure 2.

Time course of Heme-Transfer from PhuS to $\mathrm{pa}-\mathrm{HO}$ as a function of $\mathrm{pH}$. (- -) $\mathrm{pH} 6.5$; (-) 7.5; (…) 8.5; (-..-) pa-HO-G125V, pH 7.5; and (---) pa-HO-Wt with 10mM KCN, pH 7.5. Experiments were conducted with $10 \mu \mathrm{M}$ PhuS, and $30 \mu \mathrm{M} \mathrm{pa-HO}$ in $20 \mathrm{mM}$ Tris- $\mathrm{HCl}$ at the corresponding $\mathrm{pH}$ at $25^{\circ} \mathrm{C}$, and time course were measured at 405 (for $\mathrm{pH} 6.5$ and 7.5) and $410 \mathrm{~nm}(\mathrm{pH} 8.5)$. Reaction conducted in the presence of $\mathrm{KCN}$ was monitored at $419 \mathrm{~nm}$. 


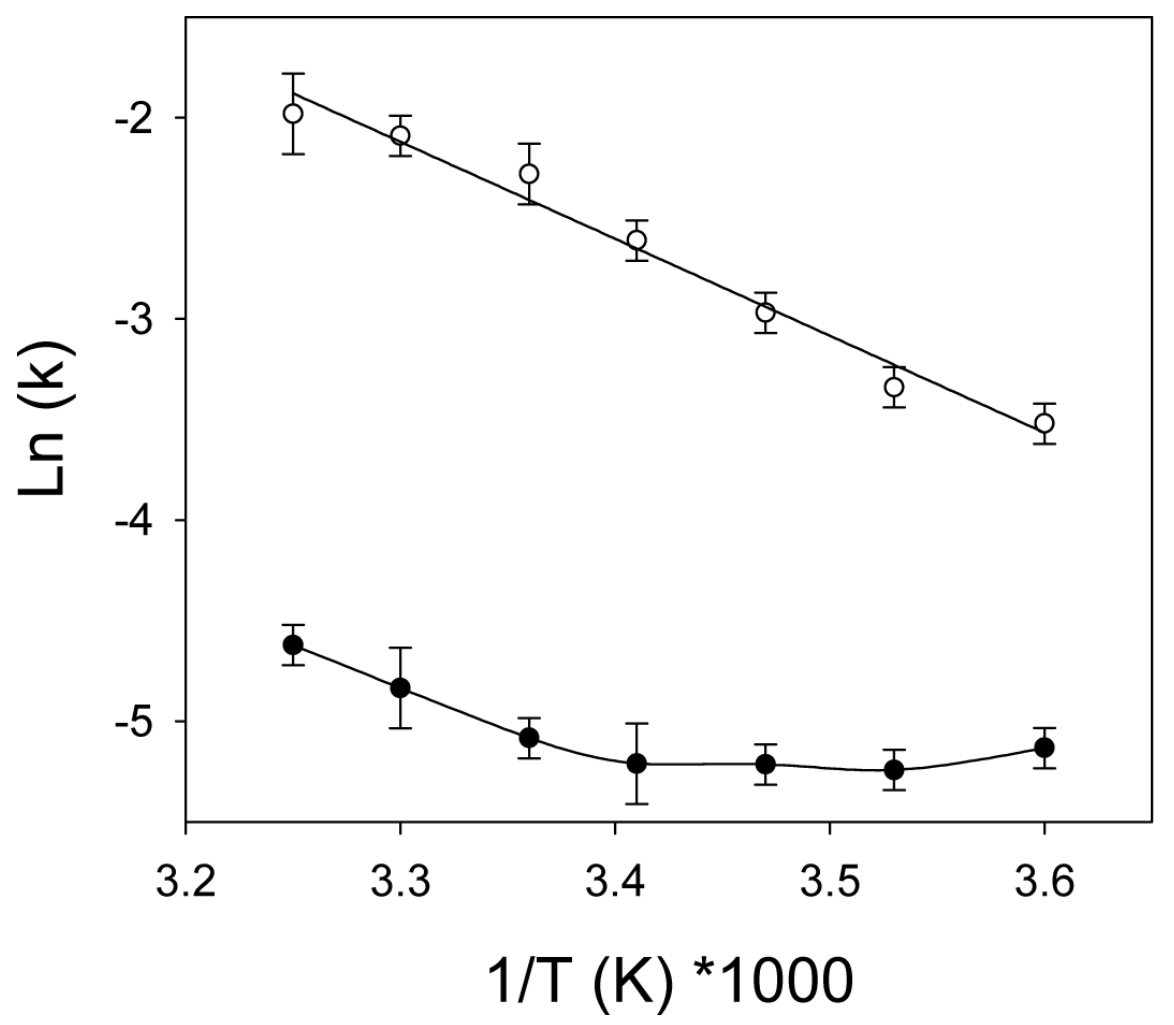

Figure 3.

Arrhenius Plot of heme transfer in the presence of $10 \mu \mathrm{M}$ PhuS and $30 \mu \mathrm{M} p a-\mathrm{HO}$ in $20 \mathrm{mM}$ Tris, pH 7.5. (o) Fast phase $\left(k_{1}\right)$ and $(\bullet)$ slow phase $\left(\mathrm{k}_{2}\right)$. 

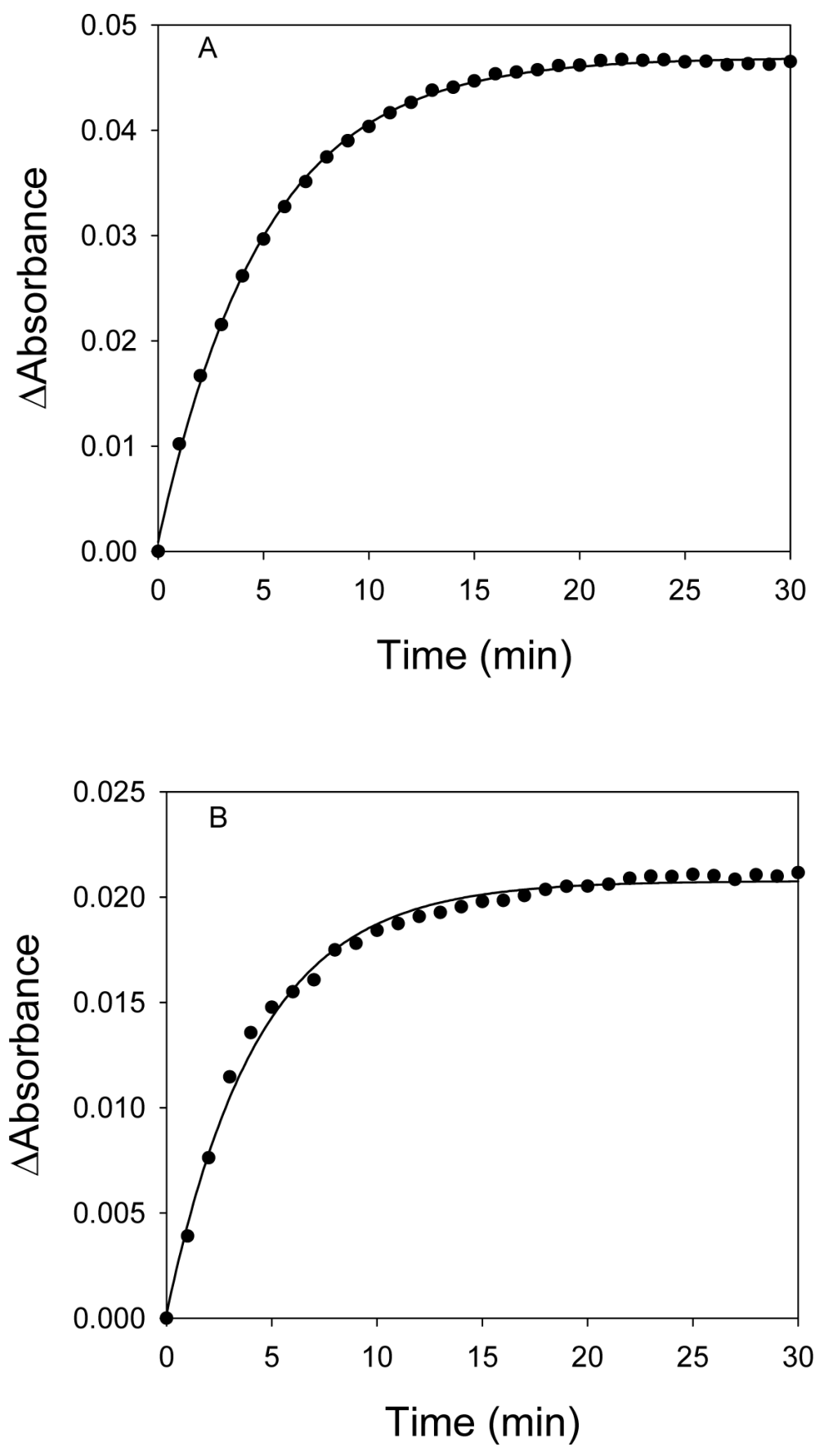

Figure 4.

Heme transfer from $p a-\mathrm{HO}$ (Panel A) and PhuS (Panel B) to myoglobin. Reactions were conducted in $20 \mathrm{mM}$ Tris- $\mathrm{HCl}, \mathrm{pH} 7.5$, at $25^{\circ} \mathrm{C}$ with $2 \mu \mathrm{M}$ pa-HO or PhuS and $12 \mu \mathrm{M}$ myoglobin and the time course was measured at $408 \mathrm{~nm}$. 
Table I

Kinetic and equilibrium parameters for heme binding to various hemeproteins.

\begin{tabular}{llll}
\hline Protein & $\boldsymbol{k}_{\mathbf{H}}\left(\mathbf{M}^{-\mathbf{1}} \mathbf{s}^{-\mathbf{1}}\right)$ & $\boldsymbol{k}_{{ }^{-} \mathbf{H}}\left(\mathbf{s}^{-\mathbf{1}}\right)$ & $\mathbf{K}_{\mathbf{d}}(\mathbf{M})$ \\
\hline Myoglobin $^{a}$ & $7.0 \times 10^{7}$ & $8.4 \times 10^{-7}$ & $1.3 \times 10^{-14}$ \\
BSA $^{-}$ & $5.0 \times 10^{4}$ & $3.2 \times 10^{-4}$ & $6.4 \times 10^{-9}$ \\
pa-HO & $1.1 \times 10^{5 c}$ & $6.6 \times 10^{-2}$ & $0.6 \times 10^{-6 d}$ \\
PhuS & $1.8 \times 10^{5 c}$ & $3.6 \times 10^{-2}$ & $0.2 \times 10^{-6 d}$ \\
\hline
\end{tabular}

${ }^{a}$ Values reported by Hargrove et al.(21), for the binding of Fe-CO to apo-myoglobin.

${ }^{b}$ Reported by Gattoni et al.(20), binding of free heme to apo-albumin

${ }^{c}$ All association rate constants $(k \mathrm{H})$ were measured using heme in $20 \mathrm{mM}$ Tris- $\mathrm{HCl}, \mathrm{pH} 8.0$ at $25^{\circ} \mathrm{C}$. The dissociation rate constants $(k$-H) were calculated from $\mathrm{K}_{\mathrm{d}}$, which is the ratio of $k-\mathrm{H}^{/ k} \mathrm{H}$.

${ }^{d} \mathrm{~K}_{\mathrm{d}}$ was obtained by fluorescence quenching and UV-Vis Spectroscopy, and is an estimate of heme affinity for the apo-protein sample. 


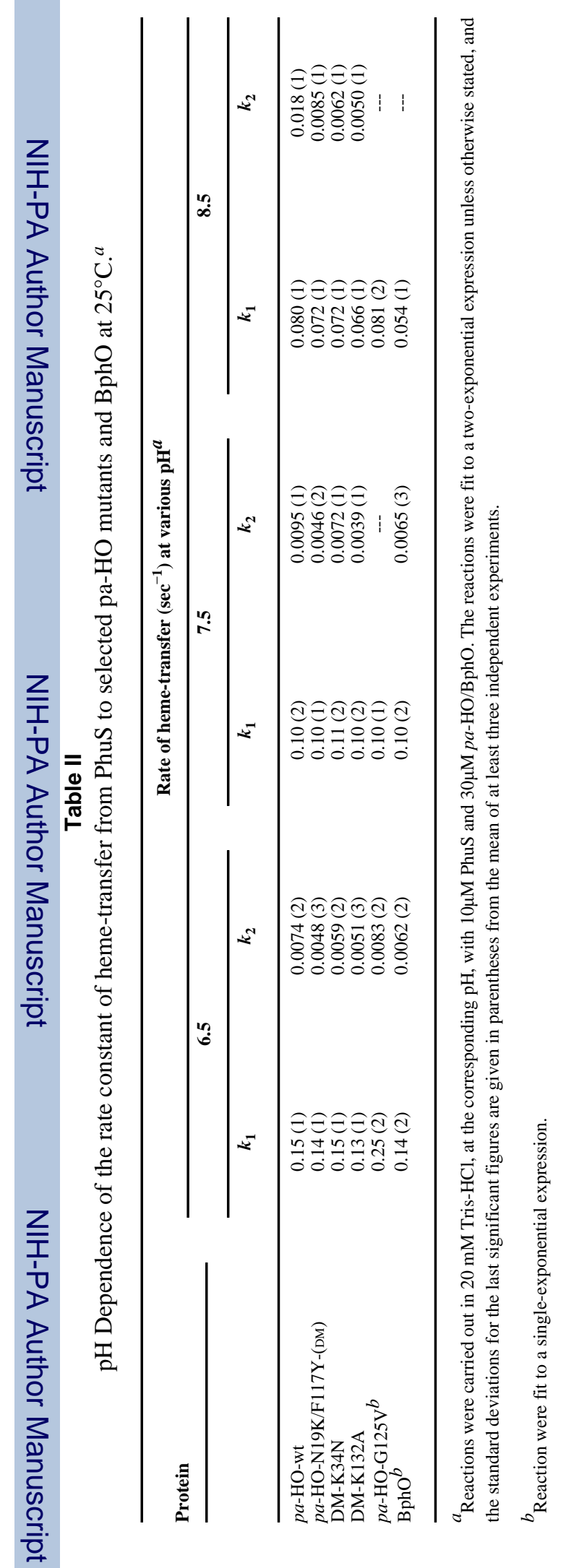

Biochemistry. Author manuscript; available in PMC 2009 January 27. 
Table III

Activation parameters for heme transfer from PhuS to pa-HO-wt, pa-HO mutants, and BphO in 20mM Tris, pH 7.5.

\begin{tabular}{|c|c|c|c|c|}
\hline Protein & $E_{\mathrm{a}}^{a}(\mathrm{kcal} / \mathrm{mol})$ & $\Delta \mathbf{H}^{\neq a}(\mathrm{kcal} / \mathrm{mol})$ & $\Delta S^{\neq a, b}\left(\mathrm{cal} \mathrm{mol}^{-1} \mathrm{~K}^{-1}\right)$ & $\Delta \mathbf{G}^{\neq}(\mathrm{kcal} / \mathrm{mol})$ \\
\hline$p a-\mathrm{HO}-\mathrm{wt}$ & 11.9 & 11.3 & -22.9 & 18.2 \\
\hline$p a-\mathrm{HO}-\mathrm{N} 19 \mathrm{~K} / \mathrm{F} 117 \mathrm{Y}-(\mathrm{DM})$ & 12.0 & 11.4 & -22.7 & 18.2 \\
\hline DM-K34N & 12.9 & 12.3 & -19.6 & 18.1 \\
\hline $\mathrm{DM}-\mathrm{K} 132 \mathrm{~A}$ & 12.2 & 11.6 & -22.1 & 18.2 \\
\hline$p a-\mathrm{HO}-\mathrm{G} 125 \mathrm{~V}$ & 12.3 & 11.7 & -22.1 & 18.3 \\
\hline $\mathrm{BphO}$ & 14.3 & 13.7 & -14.9 & 18.1 \\
\hline
\end{tabular}

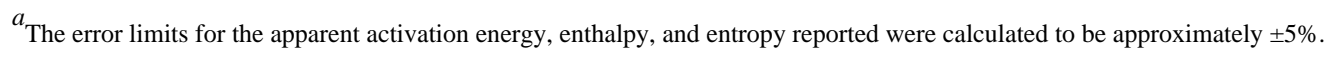

${ }^{b}$ A temperature of $298 \mathrm{~K}$ was used in the ln Eyring pre-exponential term $(\mathrm{kBT} / h)$ when it was subtracted from the ln $k$ axis intercept of the Arrhenius plot to determine the $\Delta \mathrm{S}^{\neq}$values. 
Table IV

Activation parameters for the heme transfer from PhuS to pa-HO-wt, pa-HO mutants and BphO in the presence of $10 \mathrm{mM} \mathrm{KCN}$ in $20 \mathrm{mM}$ Tris, $\mathrm{pH} 7.5$.

\begin{tabular}{|c|c|c|c|c|}
\hline Protein & $E_{\mathrm{a}}^{a}(\mathrm{kcal} / \mathrm{mol})$ & $\Delta \mathbf{H}^{\neq a}(\mathrm{kcal} / \mathrm{mol})$ & $\Delta \mathbf{S}^{\neq a, b}\left(\mathrm{cal} \mathrm{mol}^{-1} \mathbf{K}^{-1}\right)$ & $\Delta \mathbf{G}^{\neq}(\mathrm{kcal} / \mathrm{mol})$ \\
\hline $\begin{array}{l}p a-\mathrm{HO}-\mathrm{wt} \\
\text { pa-HO-N19K/F117Y-(DM) } \\
\text { DM-K34N } \\
\text { DM-K132A } \\
\text { pa-HO-G125V } \\
\text { BphO }\end{array}$ & $\begin{array}{l}14.6 \\
17.2 \\
12.3 \\
14.4 \\
14.0 \\
12.8\end{array}$ & $\begin{array}{l}14.0 \\
16.6 \\
11.7 \\
13.8 \\
13.4 \\
12.2\end{array}$ & $\begin{array}{c}-13.7 \\
-5.4 \\
-21.5 \\
-14.6 \\
-16.3 \\
-20.9\end{array}$ & $\begin{array}{l}18.1 \\
18.3 \\
18.1 \\
18.2 \\
18.2 \\
18.4\end{array}$ \\
\hline
\end{tabular}

